\title{
Increased Serum E-Selectin Levels Were Associated with Cognitive Decline in Patients with Stroke
}

This article was published in the following Dove Press journal:

International Journal of General Medicine

Jin $\mathrm{Li}^{1}{ }^{\text {** }}$

Junqiang $\mathrm{Bao}^{2, *}$

Chao $\mathrm{Gao}^{2}$

Zibin $\mathrm{Wei}^{2}$

Liguo $\operatorname{Tan}^{3}$

Ping $\mathrm{Liu}^{2}$

Zhiwei Wang ${ }^{2}$

Shujuan Tian ${ }^{2}$

'Department of Neurosurgery, The Affiliated Hospital of the Nco School, The Army Medical University, Shijiazhuang, 05004I, Hebei, People's Republic of China; ${ }^{2}$ Department of Neurology, The First Hospital of Hebei Medical University, Shijiazhuang, 050030, Hebei, People's Republic of China;

${ }^{3}$ Department of Field Internal Medicine, The NCO School of The Army Medical University, Shijiazhuang, 05003 I Hebei, China

*These authors contributed equally to this work
Correspondence: Zhiwei Wang;

Shujuan Tian

Department of neurology, The First Hospital

of Hebei Medical University, No. 89

Donggang Road, Shijiazhuang, 050030,

Hebei, People's Republic of China

Emailwzw0412@163.com;

2581810783@qq.com
Background: Previous studies have reported that patients with stroke have a high incidence of cognitive decline. The aim was to elucidate the association between serum E-selectin levels and cognitive function in stroke patients.

Materials and Methods: Serum levels of E-selectin were measured in 322 patients with stroke at baseline. Cox proportional hazard analysis was used to evaluate the predictive value of serum E-selectin for predicting cognitive decline (end point) in patients with stroke.

Results: Multivariate linear regression analysis revealed that serum E-selectin levels were independently associated with MOCA score after adjusting for age, gender, BMI, current smoker, current drinker, admission systolic and diastolic BP, CVD history and laboratory measurements in patients with stroke at baseline $(\mathrm{S} \beta=-0.156 ; 95 \% \mathrm{CI},-0.170--0.074$; $P<0.001)$. The multivariate Cox proportional hazard analysis revealed that serum E-selectin $(\mathrm{HR}=2.481,95 \%$ CI 1.533-4.327, $P$-trend $<0.001)$ was an independent prognostic factor for cognitive decline in these patients with stroke during the follow-up period.

Conclusion: Our results showed that increased serum E-selectin levels were significantly and independently associated with cognitive decline and had independent predictive value for cognitive decline in patients with stroke. Serum E-selectin might enable early recognition of cognitive decline among stroke patients.

Keywords: E-selectin, stroke cognitive decline, prognostic value

\section{Introduction}

People with stroke among older population have been strongly associated with an increased risk of developing cognitive decline. ${ }^{1-4}$ Existing studies suggested that cognitive impairment was higher in people with stroke than in those without stroke. ${ }^{5}$ Although the current mechanisms are poorly understood, increasing inflammation and oxidative stress in stroke play important impacts on an increased risk for cognitive impairment. ${ }^{6,7}$ Cognitive decline is a major health problem among older population and has been identified as the strongest independent predictor of heavy medical burden and poor quality of life. Hence, identifying relevant risk factors of cognitive decline in patients with stroke has to be addressed.

The inflammatory responses are a common feature of cardiovascular diseases (CVDs). ${ }^{8-10}$ Inflammatory mediators including interleukin (IL), chemokines and tumor necrosis factor- $\alpha(\mathrm{TNF}-\alpha)$ play an important role in the occurrence and progress of CVDs. E-selectin is an adhesion molecule and is expressed on vascular endothelial cells. Like other pro-inflammatory cytokines, such as TNF- $\alpha$ or IL-1 $\beta$, E-selectin is involved in various inflammatory reactions. ${ }^{20}$ Existing evidence suggested that E-selectin, as a new inflammatory cytokine, ${ }^{11-13}$ is involved in the 
progress of CVDs. ${ }^{14-16}$ As a chronic inflammatory disease, stroke can hasten the progression of cognitive decline and contribute to a higher rate of cognitive decline. ${ }^{4-6}$ Hence, given the close association between E-selectin and inflammation, ${ }^{11-14}$ we hypothesized that serum E-selectin levels might be associated with cognitive decline in stroke patients.

To date, few study has explored the association between serum E-selectin levels and cognitive decline in patients with stroke. We aimed to investigate whether increased E-selectin levels were associated with elevated risk of cognitive decline in patients with stroke. This was also the first study to explore the prognostic value of E-selectin for predicting cognitive decline in stroke patients.

\section{Materials and Methods}

\section{Study Population}

We studied 322 hospitalized patients from the first hospital of Hebei Medical University in China because of ischemic or hemorrhagic stroke between January 2014 and August 2016. All stroke patients did not had other serious acute illnesses and were stable for more than three months before admission. Patients with stroke were clinically stable during hospitalization and were prospectively followed up after discharge. The diagnosis of stroke was performed by two neurologists who used the same diagnostic criteria for these stroke patients. ${ }^{17}$ Montreal cognitive assessment (MOCA) was used for the assessment of mild cognitive impairment (MCI) and is a rapid screening assessment tool of cognitive function. ${ }^{18}$ The cognitive areas of assessments include concentration and attention, memory, executive function, visual structure skills, language, calculation, abstract thinking and orientation. The total score of the scale is 30 points, and patients with value $<26$ points were considered as MCI. For the purposes of this study, MCI was considered cognitive decline. Of these patients, 68 patients with stroke were identified as cognitive decline (MCI) in this study. Patients with liver and kidney dysfunction, respiratory diseases, neoplastic diseases, or other serious diseases were excluded $(\mathrm{N}=12)$.

The diagnosis of other CVDs, including coronary heart disease, hypertension and others was based on the patient's current or previous medical records. Other data on clinical characteristics, including age, gender, body mass index (BMI), smoking, alcohol consumption, blood pressure (BP) and others were collected from patient interviews or medical records. According to the Declaration of Helsinki guidelines, the Ethics Committee of the first hospital of Hebei Medical University approved this study, and all patients gave written informed consent.

\section{Follow-Up}

All patients after discharge were followed up for a mean of $34 \pm 5$ months prospectively. Patients were followed up by telephone or review of the medical record four times a year until the occurrence of MCI.

\section{Measurement of E-Selectin Levels}

Fasting venous blood samples from patients with stroke were collected within the first 24 hours after admission. The samples were prepared immediately by centrifugation and processed for determination of E-selectin levels. Serum levels of E-selectin were measured by electrochemiluminescence immunoassay (Cobas e 601, F. Hoffmann-La Roche Ltd.). E-selectin levels were measured three times, and the average of the three times was used for the final analysis (coefficient of variation of precision $[\mathrm{CV}],<5 \%$ ).

\section{Laboratory Measurements}

Fasting venous blood samples from patients with stroke were also tested for albumin (ALB), blood glucose (FBG), hemoglobin (Hb), fasting, hs-CRP (hsC-reactive protein), HbAlc (glycosylated hemoglobin), high-density lipoprotein (HDL) and LDL (low-density lipoprotein) with the use of the Siemens ADVIA 2400 automatic biochemistry analyzer (Siemens AG). Estimated glomerular filtration rate (eGFR) was calculated by the Modification of Diet in Renal Disease (MDRD) formula. ${ }^{19}$

\section{Statistical Analyses}

The Kolmogorov-Smirnov test was used to analyze the normality of the data. $P$ value $\leq 0.05$ was considered to be statistically significant. The data that were not normally distributed were analyzed by the Mann-Whitney $U$-test and then were expressed as the median (interquartile range $[\mathrm{IQR}])$. Data were analyzed by independent $t$-test and presented as the mean \pm SD for normally distributed data. The chi-square test was used to analyze analyzed categorical variables and was expressed as $\mathrm{n}(\%)$. 
Multivariate linear regression model was used to assess the independent relationship between serum E-selectin levels and MOCA score in stroke patients at baseline. Then, the Cox proportional hazard analysis was performed to assess the independent prognostic factors for cognitive decline (MCI) in patients with stroke during the follow-up period. In these Multivariate analyses, we adjusted for clinical data relevant to stroke and cognitive function even if the factors were not significantly associated with cognitive decline in the univariate analysis because they are key clinical variables and may be associated with cognitive decline in the multivariate but not univariate analyses. Additionally, sensitivity and stratification analyses were also performed to evaluate the independent association between serum E-selectin levels and cognitive decline by, respectively, adding "cognitive therapy" and "CVD history" as covariates. All of the analyses were performed by using SPSS 23.0.

\section{Results}

\section{Clinical Characteristics of Study Subjects at Baseline}

To evaluate serum E-selectin levels in stroke patients, 358 age-and gender-matched healthy subjects, who have undergone physical examination without stroke and any other severe illnesses, were obtained as the control group. Our results suggested that serum levels of E-selectin were significantly higher than healthy subjects (Table 1). The clinical characteristics at baseline of stroke patients are presented in Table 2. The age of all included patients was $65.3 \pm 5.7$ years and males were $151(46.89 \%)$. MOCA score was $25.8 \pm 4.6$ in all stroke patients.

Table I Baseline Characteristics of Patients with Stroke and Healthy Subjects

\begin{tabular}{|c|c|c|c|}
\hline Variables & $\begin{array}{l}\text { Healthy } \\
\text { Subjects } \\
(n=358)\end{array}$ & $\begin{array}{l}\text { Patients with } \\
\text { Stroke }(n=322)\end{array}$ & $P$ value \\
\hline Age (years) & $64.6 \pm 5.9$ & $65.3 \pm 5.7$ & 0.485 \\
\hline $\begin{array}{l}\text { Gender (male), } \\
\text { n (\%) }\end{array}$ & $174(48.6)$ & I5I (46.89) & 0.159 \\
\hline $\begin{array}{l}\text { Serum soluble } \\
\text { E-selectin }(\mathrm{ng} / \mathrm{mL})\end{array}$ & $31.39 \pm 4.82$ & $77.38 \pm 8.62$ & $<0.001$ \\
\hline
\end{tabular}

Note: Data are presented as mean \pm SD for normally distributed data, as median (interquartile range) for nonnormally distributed data, and as $n$ (\%) for categoric variables.
Table 2 Clinical Characteristics in 322 Patients with Stroke at Baseline

\begin{tabular}{|l|c|}
\hline Variables & $\begin{array}{c}\text { All Patients with Srokoe } \\
(\mathbf{n}=\mathbf{3 2 2})\end{array}$ \\
\hline Age (years) & $65.3 \pm 55.7$ \\
Gender (male), $\mathrm{n}(\%)$ & $151(46.89)$ \\
BMI $(\mathrm{kg} / \mathrm{m} 2)$ & $26.3(24.3-27.5)$ \\
Current smoker, $\mathrm{n}(\%)$ & $64(19.9)$ \\
Current drinker, $\mathrm{n}(\%)$ & $322(26.4)$ \\
Admission systolic BP $(\mathrm{mmHg})$ & $139.6(129.2-157.3)$ \\
Admission diastolic BP (mmHg) & $82.3(80.4-93.4)$ \\
Cognitive therapy, $\mathrm{n}(\%)$ & $31(9.6)$ \\
MOCA score & $25.8 \pm 4.6$ \\
Cognitive decline, $\mathrm{n}(\%)$ & $68(21.1)$ \\
\hline CVD history & \\
Hypertension, $\mathrm{n}(\%)$ & $234(72.6)$ \\
Coronary heart disease, $\mathrm{n}(\%)$ & $33(9.9)$ \\
Diabetes, $\mathrm{n}(\%)$ & $101(31.4)$ \\
Others, $\mathrm{n}(\%)$ & $24(7.5)$ \\
\hline Laboratory measurements & \\
eGFR (mL/min/l.73 m²) & $56.3(53.1-57.4)$ \\
Hs-CRP (pg/mL) & $6.5(2.2-11.7)$ \\
Hb (g/L) & $108(92-125)$ \\
ALB (g/L) & $34.6(32.4-44.6)$ \\
FBG (mmol/L) & $7.4 \pm 2.3$ \\
HbAlc (\%) & $5.4(4.9-6.3)$ \\
HDL (mmol/L) & $1.55 \pm 0.21$ \\
LDL (mmol/L) & $2.63 \pm 1.21$ \\
Serum soluble E-selectin $(\mathrm{ng} / \mathrm{mL})$ & $77.38 \pm 8.62$ \\
\hline
\end{tabular}

Note: Data are presented as mean \pm SD for normally distributed data, as median (interquartile range) for nonnormally distributed data, and as $\mathrm{n}(\%)$ for categoric variables.

Abbreviations: MOCA, montreal cognitive assessment; BMI, body mass index; BP, blood pressure; CVD, cardiovascular disease; eGFR, estimated glomerular filtration rate; $\mathrm{Hb}$, hemoglobin; hs-CRP, hs-C-reactive protein; ALB, albumin; FBG, fasting blood glucose on admission; HbAlc, glycosylated hemoglobin; HDL, high-density lipoprotein; LDL, low-density lipoprotein.

\section{Increased Serum Levels of E-Selectin} Showed a Significant Association with MOCA Score in 322 Patients with Stroke

To assess the association of serum E-selectin levels in stroke patients with MOCA score, multivariate linear regression model was performed (Table 3). Model 1 showed that higher serum levels of E-selectin were significantly associated with MOCA score after no adjustment was made $(\mathrm{S} \beta=-0.182,95 \% \mathrm{CI}-0.226-$ $-0.101, \mathrm{P}<0.001)$. The results of Model 2 were similar to those of Model 1 ( $\mathrm{S} \beta=-0.169,95 \%$ CI $-0.198-$ $-0.092, \mathrm{P}<0.001$ ), after adjustments were made for age, gender, BMI, current smoker, current drinker, admission systolic and diastolic BP and CVD history. This 
Table 3 Multivariate Linear Regression Analysis for the Association Between the Serum Soluble E-Selectin Levels and MOCA Score in 322 Patients with Stroke at Baseline

\begin{tabular}{|l|c|c|c|c|}
\hline Variables & $\mathbf{R}^{\mathbf{2}}$ & $\mathbf{S \beta}$ & $\mathbf{9 5 \%} \mathbf{C I}$ & $\mathbf{P}$ value \\
\hline $\begin{array}{l}\text { Serum soluble } \\
\text { E-selectin (ng/mL) } \\
\text { (per I-SD }\end{array}$ & & & & \\
increase) & & & & \\
Model I & 0.042 & -0.182 & $-0.226-0.101$ & $<0.00 \mathrm{I}$ \\
Model 2 & 0.178 & -0.169 & $-0.198--0.092$ & $<0.00 \mathrm{I}$ \\
Model 3 & 0.253 & -0.156 & $-0.170--0.074$ & $<0.00 \mathrm{I}$ \\
\hline
\end{tabular}

Notes: Model I: No adjustment. Model 2: Adjusted for age, gender, BMI, current smoker, current drinker, admission systolic and diastolic BP and CVD history. Model 3: Adjusted for age, gender, BMI, current smoker, current drinker, admission systolic and diastolic BP, CVD history and laboratory measurements.

Abbreviations: MOCA, montreal cognitive assessment; BMI, body mass index; $\mathrm{BP}$, blood pressure; CVD, cardiovascular disease.

relationship in Model 3 remained statistically significant and changed minimally after adding laboratory measurements to Model 2 ( $\mathrm{S} \beta=-0.156,95 \%$ CI $-0.170-$ $-0.074, \mathrm{P}<0.001)$.

\section{Cox Proportional Hazard Analysis for Predicting Cognitive Decline (MCl) in 322} Stroke Patients

All included stroke patients $(\mathrm{N}=322)$ were prospectively followed up for a mean of $34 \pm 5$ months. Sixty-eight of the included stroke patients have cognitive decline (MCI). To assess the risk factors for cognitive decline, multivariate Cox proportional hazard regression model was performed (Table 4). Multivariate Cox proportional hazard analysis suggested that serum levels of E-selectin ( $\mathrm{HR}=2.481,95 \%$ CI 1.533-4.327, $P$-trend $<0.001$, Model 3) were an independent prognostic factor for cognitive decline after adjustments of age, gender, BMI, current smoker, current drinker, admission systolic and diastolic BP, CVD history and laboratory measurements were made. Kaplan-Meier analysis showed that stroke patients with serum levels of E-selectin above the mean had a significantly higher rate of cognitive decline than patients with serum levels of E-selectin below the mean value (data not shown).

\section{Sensitivity and Stratification Analysis for Predicting Cognitive Decline $(\mathrm{MCl})$ in 322 Stroke Patients}

We performed an additional sensitivity analysis to evaluate the association of serum E-selectin with cognitive decline in stroke patients by adding "cognitive therapy" as a covariate (Table 5). The sensitivity analysis suggested that higher serum E-selectin levels were still independently associated with a higher risk of cognitive decline during the follow-up. Stratified analysis, by adding "CVD history" as a covariate, showed that the significant association between serum E-selectin levels and cognitive decline (MCI) in stroke patients was affected by CVD history. A significant association exists in patient with CVD history but not in patient without CVD history (Table 6).

\section{Discussion}

In this study, our results suggested that serum E-selectin levels had independent good predictive value for cognitive decline (MCI) in patients with stroke by multivariate Cox proportional hazard analysis.

E-selectin is an adhesion molecule and is expressed on vascular endothelial cells. Like other pro-inflammatory cytokines, E-selectin is involved in various inflammatory reactions. ${ }^{20}$ E-selectin is a mediator of leukocyte rolling and recruitment during infection. E-selectin plays an important role in the attachment of leukocytes to endothelial cells and the accumulation of leukocytes in inflamed tissues during infectious diseases, malignant tumors, and

Table 4 Cox Proportional Hazard Analysis for Predicting Cognitive Decline in 322 Patients with Stroke

\begin{tabular}{|l|c|c|c|}
\hline Variables & Model I & Model 2 & Model 3 \\
\hline Serum soluble E-selectin (ng/mL) & & & \\
Quartile I & 1.000 (ref.) & 1.000 (ref.) & 1.000 (ref.) \\
Quartile 2 & $1.85 I(1.353-3.5 I I)$ & $1.731(1.320-3.094)$ & $1.635(1.214-2.984)$ \\
Quartile 3 & $2.408(1.69 I-3.979)$ & $2.274(1.457-3.713)$ & $1.949(1.314-3.633)$ \\
Quartile 4 & $2.994(1.874-4.748)$ & $2.690(1.669-4.538)$ & $2.48 I(1.533-4.327)$ \\
P-trend & $<0.001$ & $<0.001$ & $<0.001$ \\
\hline
\end{tabular}

Notes: Model I: No adjustment. Model 2: Adjusted for age, gender, BMI, current smoker, current drinker, admission systolic and diastolic BP and CVD history. Model 3: Adjusted for age, gender BMI, current smoker, current drinker, admission systolic and diastolic BP, CVD history and laboratory measurements.

Abbreviations: BMI, body mass index; BP, blood pressure; CVD, cardiovascular disease. 
Table 5 Sensitivity Analysis for Predicting Cognitive Decline in 322 Patients with Stroke Using Cox Proportional Hazard

\begin{tabular}{|l|c|c|c|}
\hline Variables & Model I & Model 2 & Model 3 \\
\hline Serum soluble E-selectin (ng/mL) & & & \\
Quartile I & 1.000 (ref.) & $1.000($ ref.) & 1.000 (ref.) \\
Quartile 2 & $1.750(1.33 I-3.3 I 4)$ & $1.622(I .30 I-3.053)$ & $1.538(1.236-2.892)$ \\
Quartile 3 & $2.257(1.556-3.7 I 2)$ & $2.135(I .420-3.64 I)$ & $1.883(1.309-3.395)$ \\
Quartile 4 & $2.559(1.743-4.148)$ & $2.328(I .637-3.989)$ & $2.164(1.495-3.726)$ \\
P-trend & $<0.001$ & 0.001 & 0.008 \\
\hline
\end{tabular}

Notes: Model I: Cognitive therapy. Model 2: Adjusted for age, gender, BMI, current smoker, current drinker, admission systolic and diastolic BP, CVD history and cognitive therapy. Model 3: Adjusted for age, gender, BMI, current smoker, current drinker, admission systolic and diastolic BP, CVD history, laboratory measurements and cognitive therapy.

Abbreviations: BMI, body mass index; BP, blood pressure; CVD, cardiovascular disease.

Table 6 Stratified Analysis for Cox Proportional Hazard for Predicting Cognitive Decline in 322 Patients with Stroke

\begin{tabular}{|c|c|c|c|}
\hline Variables & Model I & Model 2 & Model 3 \\
\hline \multicolumn{4}{|l|}{ CVD history (yes) } \\
\hline Serum soluble E-selectin (per I-SD increase) & $2.325(1.73 I-3.82 I)$ & $2.120(1.619-3.649)$ & $1.958(1.593-3.493)$ \\
\hline$P$ value & $<0.001$ & $<0.001$ & $<0.001$ \\
\hline \multicolumn{4}{|l|}{ CVD history (no) } \\
\hline Serum soluble E-selectin (per I-SD increase) & $1.794(1.213-2.629)$ & $1.482(1.100-2.084)$ & $1.307(0.931-1.998)$ \\
\hline$P$ value & 0.031 & 0.083 & 0.103 \\
\hline
\end{tabular}

Notes: Model I: No adjustment. Model 2: Adjusted for age, gender, BMI, current smoker, current drinker, admission systolic and diastolic BP and CVD history. Model 3: Adjusted for age, gender, BMI, current smoker, current drinker, admission systolic and diastolic BP, CVD history and laboratory measurements.

Abbreviations: BMI, body mass index; BP, blood pressure; CVD, cardiovascular disease.

autoimmune diseases. ${ }^{20-22}$ Recently, E-selectin is also involved in the development of chronic vascular inflammation, ${ }^{23-27}$ which has been considered as an important role in promoting atherosclerosis-related diseases such as stroke and coronary heart disease. ${ }^{28-31}$ Therefore, based on existing evidence, it can be hypothesized that E-selectin plays an important role in cognitive decline (MCI) by stroke.

In the current study, we first showed that higher serum levels of E-selectin had an increased MOCA score at baseline and then had higher risk of cognitive decline after a followup of 3 years, which may be mostly or partly explained by the research about inflammatory mechanism performed in previous studies. ${ }^{28-30}$ These studies have suggested that chronic vascular inflammation caused by stroke promotes events of cognitive decline. ${ }^{23-31}$ The increased serum levels of E-selectin may be the result of the aggravation of inflammation in stroke patients, which suggested that higher serum E-selectin levels were associated with an increased risk rate of cognitive decline in stroke patients. The mechanism of chronic inflammation seems to be a better explanation of the association between E-selectin and cognitive decline, which is consistent with our results. Additionally, we performed an additional sensitivity analysis to evaluate the association of serum E-selectin with cognitive decline in stroke patients by adding "cognitive therapy" as a covariate. The results suggested that higher serum E-selectin levels were still independently associated with a higher risk of cognitive decline during the follow-up of 3 years. Therefore, the early detection of cognitive decline in these stroke patients may provide us a change to develop strategies aiming to reduce the medical burden and improve prognosis. Our results seemed to imply that serum E-selectin might be a sensitive biomarker for the early recognition of cognitive decline in patients with stroke.

Our results have several obvious strengths. First, we are first to found that serum E-selectin can be considered as a valuable prognostic factor for cognitive decline in stroke patients. Second, we ensured comprehensive follow-up and rigorous adjudication of cognitive decline (MCI) and so our results are very reliable. Third, the serum E-selectin assay chosen for this study is both widely available and 
analytically stable (coefficient of variation of precision $<5 \%$ ). This serum index is convenient and reliable for clinical popularization. Certainly, this study also has several limitations. The study size was single-center with fairly small samples. Then, E-selectin was only measured once in the morning of the first day after admission. The time courses of E-selectin levels were not evaluated. Information regarding the long-term prognosis throughout the hospitalization period was not available. Further investigation will be necessary to solidify the clinical significance of E-selectin on predicting cognitive decline in patients with stroke by evaluating the time course of E-selectin.

\section{Conclusions}

We demonstrated that serum E-selectin is an independent prognostic factor for cognitive decline in patients with stroke. These results suggest that E-selectin might be a useful biomarker for predicting cognitive decline of patients with stroke.

\section{Disclosure}

The authors report no conflicts of interest in this work.

\section{References}

1. Romá N, Stroke GC. Cognitive decline and vascular dementia: the silent epidemic of the 21st century. Neuroepidemiology. 2003;22 (3): 161 .

2. Tim M. Reader response: association of orthostatic hypotension with incident dementia, stroke, and cognitive decline. Neurology. 2019.

3. Stefanidis KB, Askew CD, Greaves K, Summers MJ. The effect of non-stroke cardiovascular disease states on risk for cognitive decline and dementia: a systematic and meta-analytic review. Neuropsychol Rev. 2018;28(1):1-5.

4. Levine DA, Wadley VG, Langa KM, et al. Risk factors for poststroke cognitive decline: the REGARDS Study (Reasons for Geographic and Racial Differences in Stroke). Stroke. 2018;49(4):987-994.

5. Hnid K. Arterial stiffness and other vasculatures impairments that cause in hypertensive older patients and smoking cognitive impairments are not linked to gender. Stroke. 2009;40(9):e539;authore reply e540.

6. Lee YH, Yeh SJ. Correlation of common carotid artery intima media thickness, intracranial arterial stenosis and post-stroke cognitive impairment. Acta Neurol Taiwan. 2007;16(4):207-213.

7. Hollocks MJ, Brookes RL, Morris RG, Markus HS. The Brief Memory and Executive Test (BMET): a cognitive screening tool to detect and differentiate vascular cognitive impairment and alzheimer's disease. Int J Geriatr Psychiatry. 2017;33(2):e273-e279.

8. Granger DN, Vowinkel T, Petnehazy T. Modulation of the inflammatory response in cardiovascular disease. Hypertension. 2004;43 (5):924-931.

9. Liu D, Zeng X, Li X, et al. Role of NLRP3 inflammasome in the pathogenesis of cardiovascular diseases. Basic Res Cardiol. 2018;113 (1):5.

10. Landry NM, Cohen S, Dixon IMC. Periostin in cardiovascular disease and development: a tale of two distinct roles. Archiv für Krslaufforschung. 2018;113(1):1.
11. Adams DH, Hubscher SG, Fisher NC, et al. Expression of E-selectin and E-selectin ligands in human liver inflammation. Hepatology. 1996;24:3

12. Noble KE, Panayiotidis P, Collins PW, et al. Monocytes induce E-selectin gene expression in endothelial cells: role of CD11/CD18 and extracellular matrix proteins. Eur J Immunol. 1996;26(12): 2944-2951.

13. Sreejayan N, Wittig BM, von Stillfried N, et al. Human biliary mucin binds to E-selectin: a possible role in modulation of inflammation. Am J Physiol. 2001;280(5):G1043-G1048.

14. Gbadoe KM, Berdouzi N, Aguiñano AA, Ndiaye NC, Visvikis-Siest S. Cardiovascular diseases-related GNB3 C825T polymorphism has a significant sex-specific effect on serum soluble E-selectin levels. J Inflamm (Lond). 2016;13:1-7.

15. Hebbar M Dr, Krzewinski-Recchi MA, Hornez L, et al. Prognostic value of tumoral sialyltransferase expression and circulating E-Selectin concentrations in node-negative breast cancer patients. Int J Biol Markers. 2018;18(2):116-122.

16. Raffray L, Giry C, Thirapathi Y, et al. Increased levels of soluble forms of E-selectin and ICAM-1 adhesion molecules during human leptospirosis. PLoS One. 2018;12(7):e0180474.

17. Phipps MS, Cronin CA. Management of acute ischemic stroke. $B M J$. 2020;368:6983. doi:10.1136/bmj.16983

18. Wong A, Law LSN, Liu W, et al. Montreal cognitive assessment. Stroke. 2015;642(4):342-349.

19. Levey AS, Stevens LA, Schmid CH, et al. A new equation to estimate glomerular filtration rate. Ann Intern Med. 2009;150 (9):604-612.

20. Darveau RP, Cunningham MD, Bailey T, et al. Ability of bacteria associated with chronic inflammatory disease to stimulate E-selectin expression and promote neutrophil adhesion. Infect Immun. 1995;63 (4):1311-1317.

21. Benekli M, Güllü IH, Tekuzman G, et al. Circulating intercellular adhesion molecule-1 and E-selectin levels in gastric cancer. $\mathrm{Br}$ J Cancer. 1998;78(2):267-271.

22. Mcmurray RW. Adhesion molecules in autoimmune disease. Semin Arthritis Rheum. 1996;25(4):215-233.

23. Lv Y, Kim K, Sheng Y, et al. YAP controls endothelial activation and vascular inflammation through TRAF6. Circ Res. 2018;123 (1):43-56.

24. Tsoref O, Tyomkin D, Amit U, et al. E-selectin-targeted copolymer reduces atherosclerotic lesions, adverse cardiac remodeling, and dysfunction. J Control Release. 2018;288:136-147.

25. Smith ML, Olson TS, Ley K. CXCR2- and E-selectin-induced neutrophil arrest during inflammation in vivo. J Exp Med. 2004;200 (7):935-939.

26. Gotoh R, Suzuki J, Kosuge H, et al. E-selectin blockade decreases adventitial inflammation and attenuates intimal hyperplasia in rat carotid arteries after balloon injury. Arterioscler Thromb Vasc Biol. 2004;24(11):2063-2068.

27. Tonetti MS, D'Aiuto F, Nibali L, et al. Treatment of periodontitis and endothelial function. $N$ Engl J Med. 2007;356(9):911-920.

28. Clouston SAP, Richmond LL, Scott SB, et al. Pattern recognition to objectively differentiate the etiology of cognitive decline: analysis of the impact of stroke and alzheimer's disease. Neuroepidemiology. 2020;54(6):1-8.

29. Tang EY, Amiesimaka O, Harrison SL, et al. Longitudinal effect of stroke on cognition: a systematic review. $J$ Am Heart Assoc. 2018;7 (2): $\mathrm{e} 006443$.

30. Mattaceraso FUS, Cammen TJMVD, Hofman A, et al. Arterial stiffness and risk of coronary heart disease and stroke: the Rotterdam Study. Circulation. 2006;113(5):657-663.

31. Kaptoge S, Di Angelantonio E, Lowe G, et al.; Emerging Risk Factors Collaboration. C-reactive protein concentration and risk of coronary heart disease, stroke, and mortality: an individual participant meta-analysis. Lancet. 2010;375(9709):132-140. 


\section{Publish your work in this journal}

The International Journal of General Medicine is an international, peer-reviewed open-access journal that focuses on general and internal medicine, pathogenesis, epidemiology, diagnosis, monitoring and treatment protocols. The journal is characterized by the rapid reporting of reviews, original research and clinical studies across all disease areas. The manuscript management system is completely online and includes a very quick and fair peer-review system, which is all easy to use. Visit http://www.dovepress.com/ testimonials.php to read real quotes from published authors.

Submit your manuscript here: https://www.dovepress.com/international-journal-of-general-medicine-journal 